Acta Cryst. (2002). A58 (Supplement), C23

RECENT NMR AND MUSR RESULTS ON PHASE SEPARATION IN MANGANITES

R. De Renzi

Dipartimento di Fisica e Unita' INFM di Parma, Italy

Manganites are far from being a simple magnetic system. They provide both complex magnetic structures and an excitation spectrum not yet fully resolved. This is true of the inhomogeneous low-doping part of their phase diagram, but also of the rational fractional compositions, such as $\mathrm{La}_{0.5} \mathrm{Ca}_{0.5} \mathrm{MnO}_{3}$, and, for specific aspects, even of the end member $\mathrm{LaMnO}_{3}$. A local probe, like the nuclear or the muon spin, detects both static order and its excitations. The spin is subject to magnetic couplings, but nuclei with spin $I>1 / 2$ are also sensitive to electric field gradients, coupled to their electric quadrupole moment. A local probe has limited sensitivity in q-space (but non zero!). One specific advantage is the relatively long time-window, i.e. access to very low energy excitations, which complements the typical energy scales and resolutions of scattering. In simple magnetic systems there is a standard direct correspondence between NMR, MuSR and, say, neutron scattering results. After a brief review of the principles a number of examples shall be offered to illustrate our findings on manganites, including a few controversial situations. Among the others: how muons and neutron scattering complement each other in determining the phase diagram of $\mathrm{La}_{1-\mathrm{x}} \mathrm{Sr}_{1+\mathrm{x}} \mathrm{MnO}_{4}$; how the slow polaron dynamics may be detected and how its peculiar nature is determined comparing muons and NMR; how orbital order affects magnetism in a simpler insulating system $\left(\mathrm{KCuF}_{3}\right)$; how MuSR, NMR and neutron scattering may detect a different ordered moment.

Keywords: MANGANITES, NMR, MUSR

Acta Cryst. (2002). A58 (Supplement), C23

\section{MAGNETIC COMPTON SCATTERING STUDY OF DOUBLE} PEROVSKITE $\mathrm{Sr}_{2} \mathrm{FeMOO}_{6}$

$\underline{\text { A. Deb }}^{1}$ N. Hiraoka ${ }^{2}$ M. Itou ${ }^{3}$ Y. Sakurai ${ }^{4}$ A. Koizumi ${ }^{5}$ Y. Tomioka ${ }^{6}$ Y. Tokura $^{7}$

${ }^{1}$ Japan Synchrotron Radiation Research Institute(JASRI), SPring- ${ }^{2}$ Japan Synchrotron Radiation Research Institute(JASRI), SPring- ${ }^{3}$ Japan Synchrotron Radiation Research Institute(JASRI), SPring- ${ }^{4}$ Japan Synchrotron Radiation Research Institute(JASRI), SPring- ${ }^{5}$ Himeji Institute of Technology ${ }^{6}$ JRCAT/AIST and CERC/AIST ${ }^{7}$ JRCAT/AIST, CERC/AIST, University of Tokyo

The double perovskite, $\mathrm{Sr}_{2} \mathrm{FeMoO}_{6}$ draws fundamental and technological attention since the room-temperature magnetoresistance has been reported. It has been reported that the crystallographic data do not indicate any substantial Jahn-Teller distortion and the lattice does not appear to play any important role in this compound. A density functional calculation has predicted that this compound has a high spin-polarization of conduction carriers because of its half-metallic nature. The density of states of the down-spin band (occupied by both the Mo $\mathrm{t}_{2 \mathrm{~g}}$ and $\mathrm{Fe} \mathrm{t}_{2 \mathrm{~g}}$ electrons) is present at the Fermi level, while the upspin band (mainly composed of Fe 3 d electrons) forms a gap at the Fermi level. In this compound, hence, the relation of the orbital occupation and the magnitude of spin polarization is of interest, which seems to be relevant to ferrimagnetic half-metallic feature. A magnetic Compton scattering experiment has been performed recently on single crystals of $\mathrm{Sr}_{2} \mathrm{FeMoO}_{6}(\mathrm{Fe} / \mathrm{Mo}$ ordering of about $92 \%$ ) in the high-energy inelastic scattering beamline BL08W at SPring-8. The Magnetic Compton profiles (MCP's) were measured at $\mathrm{T}=10 \mathrm{~K}$ and $300 \mathrm{~K}$ along the [100] and [110] crystallographic directions, in which the down-spin contribution is unambiguously observed as a large dip below $\mathrm{p}_{z}=1.0$ atomic units (a.u.) in the MCP's. Besides at $\mathrm{T}=10 \mathrm{~K}$, a small peak appears at $\mathrm{p}_{\mathrm{z}}=0$ a.u. in the MCP along the [100] direction, which may have been due to the band dependent spin fluctuation.

Keywords: COMPTON PROFILE, MOMENTUM DENSITY, SYNCHROTRON RADIATION
Acta Cryst. (2002). A58 (Supplement), C23

PHASE SEPARATION SCENARIO IN THE $\mathrm{La}_{5 / 8-\mathrm{y}} \mathrm{Pr}_{\mathrm{y}} \mathrm{Ca}_{5 / 8} \mathrm{MnO}_{3}$ SERIES BY SYNCHROTRON AND NEUTRON DIFFRACTION M.A.G. Aranda ${ }^{1}$ J.A. Collado ${ }^{1}$ C. Frontera ${ }^{2}$ J.L. Garcia-Munoz ${ }^{2}$

${ }^{1}$ Universidad de Malaga, Spain ${ }^{2}$ ICMAB-CSIC, Barcelona, Spain

Nanoscopic and mesoscopic phase separation is at the forefront of theory and experiments in magnetic-conductors material science. $\mathrm{La}_{5 / 8-\mathrm{y}} \mathrm{Pr}_{\mathrm{y}} \mathrm{Ca}_{3 / 8} \mathrm{MnO}_{3}$ is a paradigmatic series, which displays a rich phase diagram at low temperatures (LT) going from metallic $(\mathrm{y}=0)$ to semiconducting $(\mathrm{y}=5 / 8)$ for the same doping level. It is known that phase separation occurs in this family, although several scenarios are under debate. We have collected T-variable powder diffraction data for $\mathrm{y}=0,0.20,0.35$ and 5/8, SXRPD (BM16, ESRF) to follow the phase(s) evolution and NPD (D2B, ILL) to assign/determine their magnetic structure(s). $\mathrm{La}_{5 / 8} \mathrm{Ca}_{3 / 8} \mathrm{MnO}_{3}$ does not undergo any structural phase transition on cooling and the M-I transition at $270 \mathrm{~K}$ is due to the ferromagnetic ordering. Semiconducting $\mathrm{Pr}_{5 / 8} \mathrm{Ca}_{3 / 8} \mathrm{MnO}_{3}$ undergoes a phase transition on cooling at 220 K from orthorhombic Pnma to monoclinic Pm. NPD data are fully compatible with the SXRPD data showing only ferromagnetic (F) peaks for $\mathrm{y}=0$ and only anti-ferromagnetic (AF) peaks for $\mathrm{y}=5 / 8 . \mathrm{La}_{0.275} \mathrm{Pr}_{0.35} \mathrm{Ca}_{3 / 8} \mathrm{MnO}_{3}$ undergoes a mesoscopic phase separation on cooling as measured by SXRPD with a M-I transition at $110 \mathrm{~K}$. NPD data showed the coexistence of F and AF peaks with a unique nuclear structure within its limited resolution. In this work, we focus on the evolution of the separated phases with temperature, a key issue to understand the real mechanism governing the metal-insulator transition and the phase separation phenomena for the intermediate compositions. We will present chemical, structural and microstructural data for this series to distinguish between possible scenarios.

\section{Keywords: PHASE TRANSITION CHARGE ORDERING CMR}

Acta Cryst. (2002). A58 (Supplement), C23

SYNTHESIS AND STRUCTURAL STUDIES OF A NEW PEROVSKITE SYSTEM : $\mathrm{Pb}(\mathrm{Bi}) \mathrm{MnO}_{3}$

C. Bougerol M.F. Gorius P. BordetI.E. Grey

Laboratoire de Cristallographie CNRS-UJF, BP 166, 38042 Grenoble cedex 9, France

The discovery of colossal magneto-resistance effects in doped $\mathrm{LaMnO}_{3}$ has led to an intense research activity on manganese-based oxides, including the search for new phases. Recently, the effect of $\mathrm{Sr}$ substitution for $\mathrm{Bi}$ in the ferromagnetic perovskite $\mathrm{BiMnO}_{3}$ has been investigated, on a structural as well as magnetic point of view [Chiba et al. JSSC 132, 139, 1997; Atou et al. JSSC $145,639,1999]$. As the $6 \mathrm{~s} 2$ lone pair of $\mathrm{Bi}^{3+}$ likely plays an important role on the structural distortion, we have decided to look for the substitution of $\mathrm{Bi}$ by a divalent cation having a lone pair, $\mathrm{Pb}^{2+}$. By using high pressure-high temperature synthesis, we have obtained the solid solution $\mathrm{Bi}_{1-\mathrm{x}} \mathrm{Pb}_{\mathrm{x}} \mathrm{MnO}_{3}$, down to $\mathrm{PbMnO}_{3}$ which had never been reported before. From $\mathrm{x}=0$ to $\mathrm{x}=0.7$, the structural arrangement belongs to the cubic perovskite type, with symmetry changes from monoclinic to tetragonal. On the other hand, $\mathrm{PbMnO}_{3}$ has a hexagonal perovskite type structure. Furthermore, changing the oxygen stoichiometry induces a modification of the layers stacking of this compound. Results about the structure of the different phases as well as magnetic properties will be presented.

\section{Keywords: PEROVSKITE, MAGNETISM,MANGANITES}

